\title{
The prognosis of autotransplanted tooth on molar region: 5 years follow up cases
}

\author{
Yong Yoon, Yong-Gun Kim, Jo-Young Suh, and Jae-Mok Lee* \\ Department of Periodontics, College of Dentistry, Kyungpook National University, Daegu 41940, Republic of Korea
}

(Received Jun 5, 2017; Revised version received Sep 5, 2017; Accepted Sep 6, 2017)

\begin{abstract}
Tooth autotransplantation is the transplantation of embedded, impacted, or erupted teeth from one site to surgically prepared sockets or extraction sites within the same individual. Tooth autotransplantation is a valuable method to replace missing teeth, instead of other procedures like dental implants or prosthetic replacements. Discrimination of indication and consideration of delicate factors affecting the results is important to success in the procedure. This study reports a 5 year clinical observation of two different cases.
\end{abstract}

KEY WORDS: Autologous, Periodontal diseases, Prognosis, Transplantation

\section{Introduction}

Tooth autotransplantation is defined as the transplantation of embedded, impacted, or erupted teeth from one site to another in the same individual, into surgically prepared socket or extraction sites [1]. According to recent prospective studies, the overall 1-year survival rate of tooth autotransplantation was $96 \%$ and the 5-year survival rate was $84 \%$ [2].

This is a possible option for the replacement of missing or extracted permanent tooth, for example, in cases of congenital tooth agenesis, malformation, unrestorable caries, or traumatic injuries, periodontal disease, ectopic eruption, and endodontic failure $[3,4]$. Autotransplantation is a general procedure in dentistry for replacing a missing tooth or extraction. Benefit of autotransplantation is that implant-supported prosthesis or other prosthetic treatment is not needed. Moreover, the transplanted tooth can serve as a normal tooth when the procedure is successful [2].

\section{*Corresponding author: Jae-Mok Lee}

Department of Periodontics, College of Dentistry, Kyungpook National University, 2175 Dalgubeol-daero, Jung-gu, Daegu 41940, Republic of Korea

Tel.: +82-53-600-7501, Fax: +82-53-427-3263

E-mail: leejmperio@gmail.com
Therefore, in addition to improved esthetics and mastication, successful tooth transplantation offers arch space maintenance and preserves the volume and morphology of the alveolar bone $[5,6]$. The cost is also considerably low in comparison to advanced treatment options such as dental implants and/or prosthetic replacements. Moreover, it can be performed as a single-step surgical procedure. Provided that case selection as carried out meticulously, autotransplantation can serve as a realizable, fast and economical treatment option for patients [7]. The procedure involves atraumatic removal of a donor tooth, creation of a socket at the recipient site, and replantation of the tooth into the new position. In mature teeth, root canal therapy is also necessary [8].

In spite of broad use of dental implants, autogenous tooth transplantation is sometimes difficult. A multitude factors influence to the success rate: including developmental stage of the root, type of tooth, surgical trauma, the time tooth is preserved outside of the alveolar bone, the shape/ site of the recipient socket, and vascularity of the recipient bed.

The outcome of the autotransplantation depends on deliberate choice of case, delicate surgical technique and an understanding of the biologic principle. Conditions of the recipient site are diverse, depending on the diagnosis of the tooth. The success rate of autotransplantation is 
Yong Yoon et al.

affected by age of patients, developmental stage of donor tooth, surgical technique without any trauma during extraction and storage of donor tooth [9]. The factors affecting the results of tooth autotransplantation have been reported in previous studies $[10,12,13]$.

The aim of the present study is to present two clinical cases of tooth autotransplantation and to assess the prognosis of tooth autotransplantation on molar region.

\section{Case Report}

\section{Case 1}

A 35-year-old, nonsmoking and not medically compromised female patient presented severe bone loss and mobility degree 3 in maxillary left first molar (Fig. 1). After extraction of the tooth, clinical and radiographic examinations showed the suitability of both donor tooth (maxillary right third molar) (Fig. 2) and recipient site.

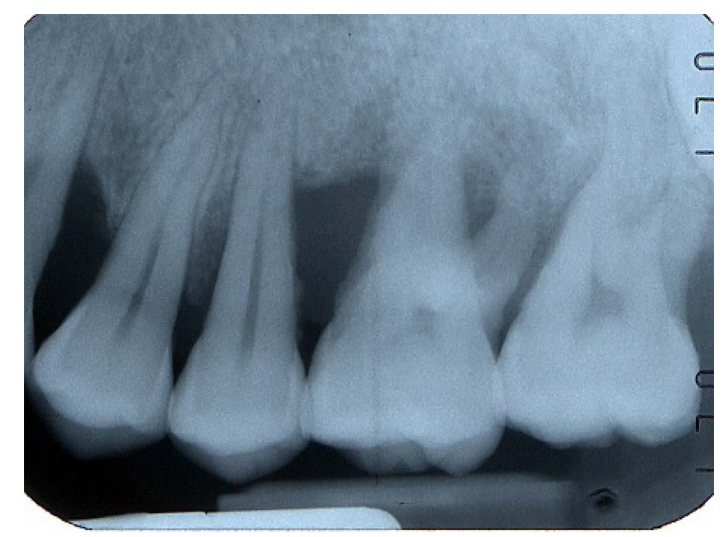

Fig. 1. Initial radiograph, showing severe bone resorption of maxillary left first molar. The tooth was diagnosed hopeless and was planed extraction and tooth autotransplantation.
Maxillary right third molar was transplanted and surgical wound was sutured (Fig. 3). Root canal therapy was carried out 2 weeks after autotransplantation. After the endodontic treatment was completed, a temporary tooth was given. Confirming that there was almost no mobility of the tooth and no problem in mastication, the permanent prosthesis was set after the one month of the treatment.

One year later, patient complained of pain associated with left mandibular second molars and the mobility of the, so the tooth was extracted.

The patient was followed after one week, two weeks, nine months (Fig. 4), three years (Fig. 5), and five years (Fig. 6). Radiographic and clinical evaluation of maxillary autotransplanted tooth satisfactory tooth stability and healthy gingival condition.

\section{Case 2}

A 34-year-old, smoking and not medically compromised male patient presented extraction of mandibular right sec-

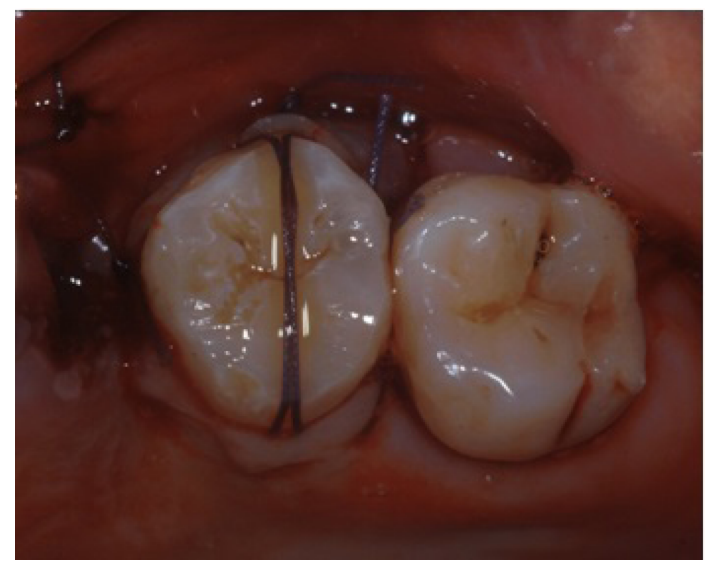

Fig. 3. Clinical photograph after tooth autotransplantation. The autotransplanted tooth was stabilized by sutures.

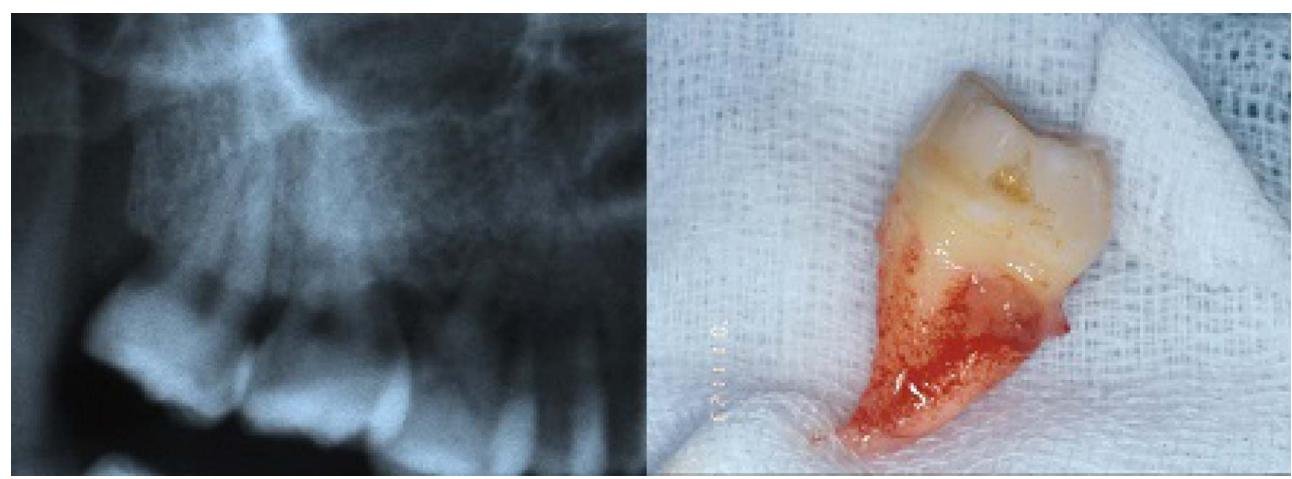

Fig. 2. Periapical radiograph of donor maxillary right third molar and clinical photograph of donor tooth after extraction. The donor tooth was carefully removed, taking precautions not to injure the root surface, and was placed in cool sterile saline while the recipient site was prepared. 


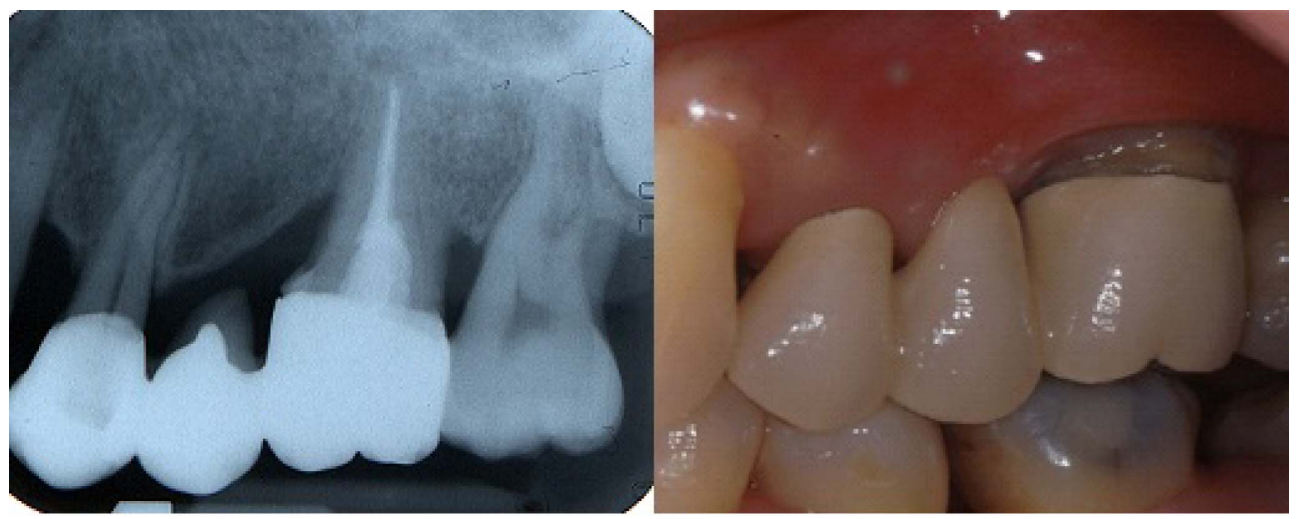

Fig. 4. Periapical radiogragh and intraoral view nine months postsurgery. No signs of root resorption in the transplanted tooth.

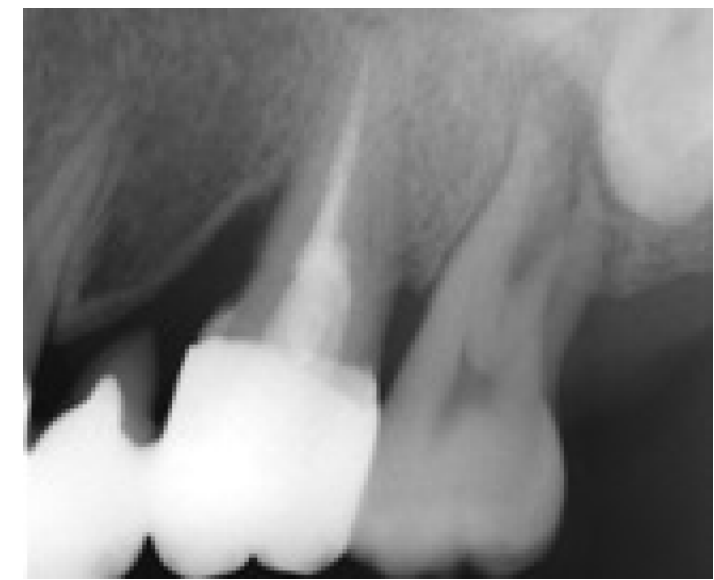

Fig. 5. Periapical radiograph three years postsurgery. No signs of additional root or bone resorption in the transplanted tooth.

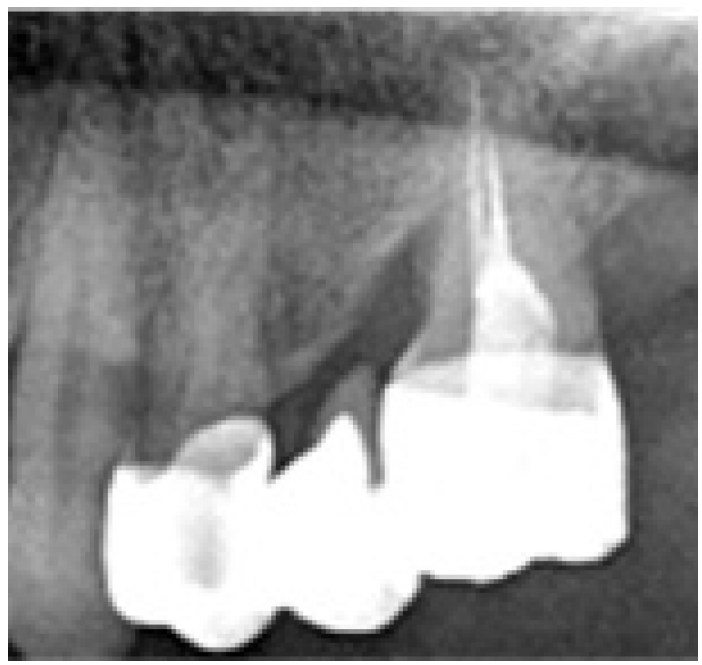

Fig. 6. Periapical radiograph five years postsurgery. The transplanted tooth was normal in the clinical and radiographic exam.

ond molar and autotransplantation of mandibular right third molar (Fig. 8) to the extraction socket was planned

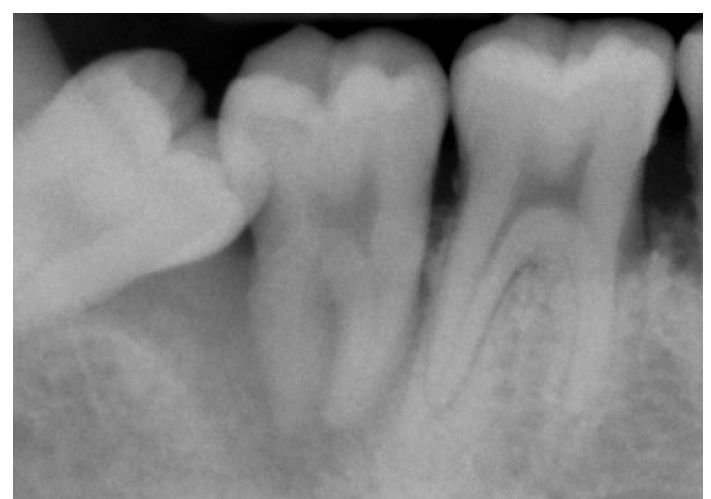

Fig. 7. Initial radiograph showing distal bone resorption of right mandibular second molar. The tooth could not be saved and it was decided to transplant the adjacent third molar to take its place.

(Fig. 9). Mandibular right second molar revealed severe bone resorption (Fig. 7). After tooth autotransplantation, clinical and radiographic examinations were followed (Fig. 10 and 11). Radiographic examination after ten months presented healing uneventful (Fig. 12). Clinical and radiographic examination after five years showed gingival swelling and bone resorption (Fig. 13). There were severe bone resorption and pathologic periodontal signs (deep pocket depth) after seven years. Finally, autotransplanted tooth was extracted.

In both cases, endodontic treatment was performed at 2 weeks after the tooth autotransplant. It was recommended that endodontic treatment be started within 7-10 days after tooth transplantation in order to prevent a pulpal origin infection [15].

\section{Discussion}

Autotransplantation of teeth provide a new treatment 
Yong Yoon et al.

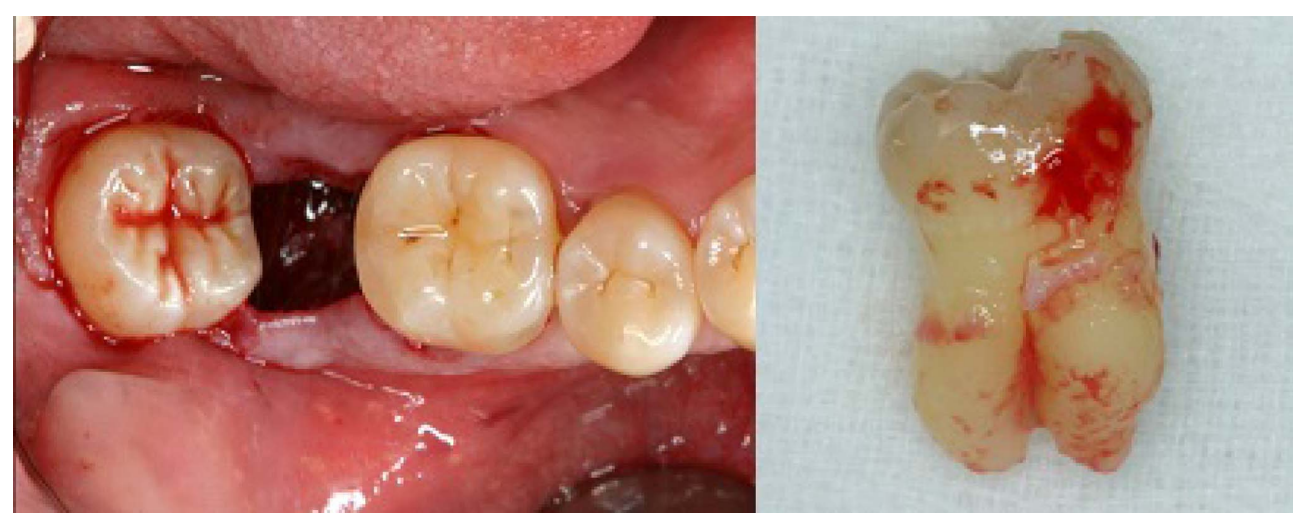

Fig. 8. Clinical photograph of recipient site and donor mandibular right third molar. Before preparing the recipient socket, the donor tooth should be extracted and examined for anatomical form, size and PDL condition.

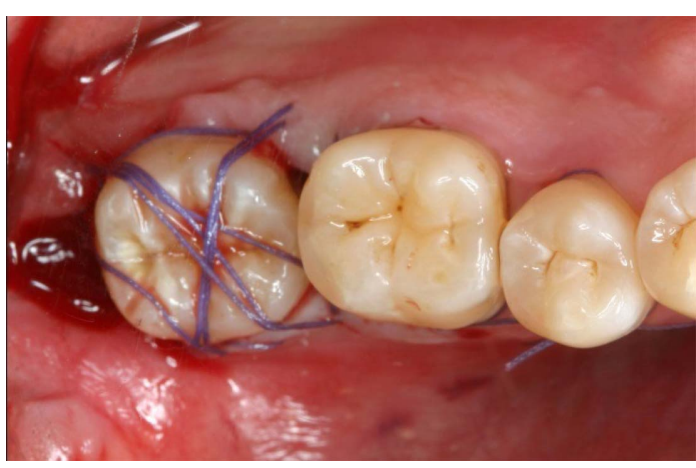

Fig. 9. The tooth was transplanted and splinted with sutures. It allows physiological mobility of the transplanted tooth.

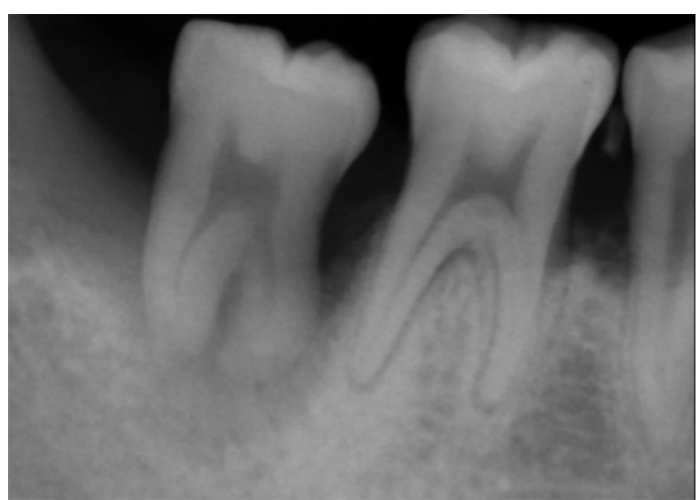

Fig. 10. Periapical radiograph reveals incomplete healing stage of transplanted tooth two weeks after tooth autotransplantation.

choice for some clinical cases. Moreover, advantages of tooth autotransplantation is better alternative than fixed or removable prosthodontics. Autotransplantation of a third molar is sometimes an appropriate alternative for replacement of a first or second molar, with conventional prosthetic treatment of implant restoration [9]. The costs for tooth transplantation are much less that for implant resto-

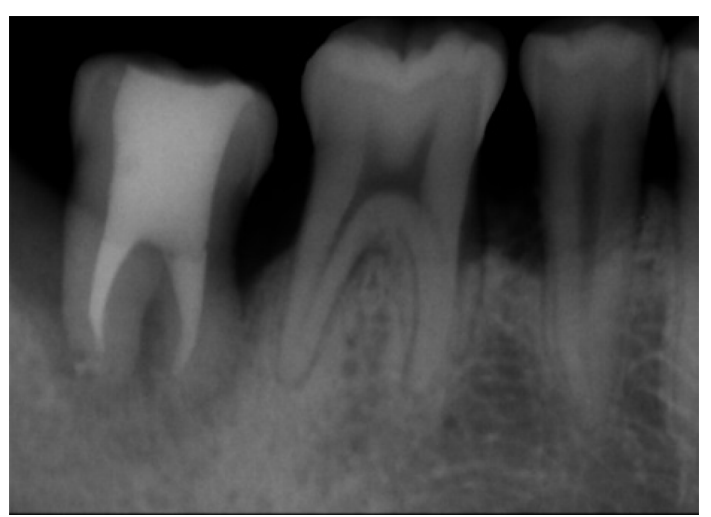

Fig. 11. Periapical radiograph presents root canal treatment two months after tooth autotransplantation.

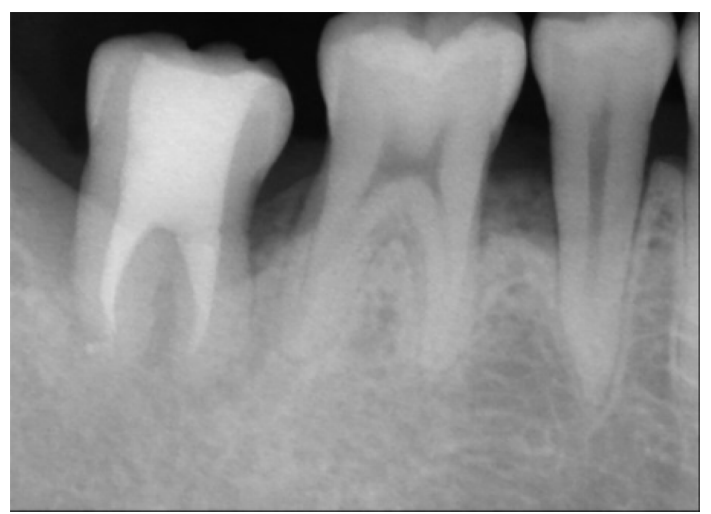

Fig. 12. Periapical radiograph of transplanted tooth ten months after tooth autotransplantation. It can be observed to be reduced apical radiolucency on distal root area of the transplanted tooth.

ration or crown and bridge prosthetic treatment.

Studies on transplanted teeth have shown teeth with incomplete root formation would undergo revascularization. Moreover, patients of young ages are usually considered to be more suitable candidates for tooth transplantation [6]. 


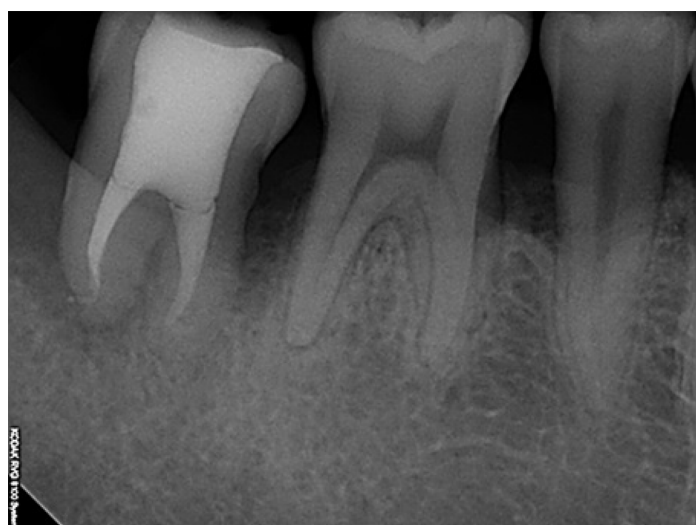

Fig. 13. Periapical radiograph of transplanted tooth five years after tooth autotransplantation. Note the distal bone resorption of the transplanted third molar.

The reason for initial infection of the autotransplanted tooth could be due to unskilled surgical technique or inappropriate surgical procedure. The presence of entire and undamaged periodontal ligament tissue on the root surface of the donor tooth is the most important factor that affects the healing of an autotransplanted teeth [12]. The extraoral time of donor tooth significantly affects the postsurgical healing [13]. The extraoral time in these cases were in 15 minutes, which was not noticeably long. Another possible reason for lost tooth may be poor adaptability between the recipient bed and donor tooth [14]. It may not be provided insufficient blood supply.

In general, the most common reason for failure of the tooth autotransplantation is root resorption. Root resorption and ankylosis is strongly related to damage to the root surface during surgical procedure and infection of the pulp [10,11]. Infected dental pulp tissue is the primary reason of a failure of transplantation to heal. To prevent the risk of these infections, it is recommended that the endodontic treatment be preceded. In this study, endodontic treatment was applied to all the patients within 2 weeks. Blood clots were observed just after the transplantation, and within 3 days after transplantation, macrophages migrated to the wound area following the formation of granulation tissue, which indicated the latter stage of inflammation. Moreover, on the 7 th day, connective tissue adherence on the root surface was shown. So it is advisable that the endodontic treatment perform after 7-10 days for initial stabilization $[15,16]$.

Implant procedures are becoming more common, concern to save natural tooth seems to be decreased. As seen in long-term follow up of tooth autotransplantation, it is a very valuable method to replace missing teeth. However, tooth autotransplantation is not recommended for patients with poor oral hygiene. Transplanted teeth have the same risks as natural teeth with caries and periodontal disease. Therefore, periodic follow up and supportive treatment should be carried out. Maintenance and good compliance of patients are necessary for satisfactory long-term results.

Careful consideration to minimize damage the periodontal ligament should be given during extraction and positioning of donor tooth. Moreover, extraoral time of donor tooth must be reduced as possible. It should be studied the preserving solution of donor tooth and surgical technique for complete healing of transplanted tooth.

Previous studies have reported a 5-year survival rate of $84 \%$ after tooth autotransplantation. Each autotransplanted tooth of both cases survived up to five years in this study. One of the patients in this study reported that the mobility of transplanted tooth was increased and felt discomfort during mastication after seven years later. As a result, extraction of the tooth was performed. In this study, tooth autotransplantation was performed at the site of bone loss due to moderate or higher periodontal disease. In the second case where a severe bone loss to the apical region was observed, the autotransplanted tooth was unstable after more than 5 years. Therefore, additional studies on the long-term prognosis of tooth autotransplantation depending on the extent and the shape of the bone loss in the recipient site will be necessary.

\section{Acknowledgements}

Persons or institutes that contributed to the papers but not enough to be coauthors may be introduced. Financial support including foundations, institutions, pharmaceutical and device manufacturers, private companies, and intramural departmental sources should be described along with other types of support, if any.

\section{Conflict of Interest}

The authors declare that they have no competing interests.

\section{ORCID}

Yong Yoon 0000-0001-9348-1410 
Yong Yoon et al.

$\begin{array}{ll}\text { Yong-Gun Kim } & 0000-0002-2793-7667 \\ \text { Jo-Young Suh } & 0000-0002-3515-3854 \\ \text { Jae-Mok Lee } & 0000-0002-0291-6114\end{array}$

\section{References}

1. Natiella JR, Armitage JE, Greene GW. The replantation and transplantation of teeth. A review. Oral Surg Oral Med Oral Pathol 1970;29:397-419.

2. Sugai T, Yoshizawa M, Kobayashi T, Ono K, Takagi R, Kitamura N, Okiji T, Saito C. Clinical study on prognostic factors for autotransplantation of teeth with complete root formation. Int J Oral Maxillofac Surg 2010;39: 1193-1203. doi: 10.1016/j.ijom.2010.06.018.

3. Kahnberg KE. Autotransplantation of teeth (I). Indications for transplantation with a follow-up of 51 cases. Int J Oral Maxillofac Surg 1987;16:577-585.

4. Kokich VG, Crabill KE. Managing the patient with missing or malformed maxillary central incisors. Am J Orthod Dentofacial Orthop 2006;129:S55-S63. doi: 10.1016/ j.ajodo.2005.11.007.

5. Thomas S, Turner SR, Sandy JR. Autotransplantation of teeth: is there a role? Br J Orthod 1998;25:275-282. doi: 10.1093/ortho/25.4.275.

6. Mendes RA, Rocha G. Mandibular third molar autotransplantation--literature review with clinical cases. J Can Dent Assoc 2004;70:761-766.

7. Azevedo PC, Moura CC, Zanetta-Barbosa D, Bernadineli N. Time of endodontic treatment in autogenic transplants of mature teeth: histological study in dogs. Oral Surg Oral Med Oral Pathol Oral Radiol Endod 2007;104:287-293. doi: 10.1016/j.tripleo.2007.02.006.
8. Patel S, Fanshawe T, Bister D, Cobourne MT. Survival and success of maxillary canine autotransplantation: a retrospective investigation. Eur J Orthod 2011;33:298304. doi: 10.1093/ejo/cjq071.

9. Mejàre B, Wannfors K, Jansson L. A prospective study on transplantation of third molars with complete root formation. Oral Surg Oral Med Oral Pathol Oral Radiol Endod 2004;97:231-238. doi: 10.1016/S1079-2104(03) 00461-X.

10. Tsukiboshi M. Autotransplantation of teeth: requirements for predictable success. Dent Traumatol 2002;18:157-180. doi: 10.1034/j.1600-9657.2002.00118.x.

11. Andreasen JO, Paulsen HU, Yu Z, Bayer T, Schwartz O. A long-term study of 370 autotransplanted premolars. Part II. Tooth survival and pulp healing subsequent to transplantation. Eur J Orthod 1990;12:14-24.

12. Andreasen JO. Interrelation between alveolar bone and periodontal ligament repair after replantation of mature permanent incisors in monkeys. J Periodontal Res 1981; 16:228-235. doi: 10.1111/j.1600-0765.1981.tb00970.x.

13. Hupp JG, Mesaros SV, Aukhil I, Trope M. Periodontal ligament vitality and histologic healing of teeth stored for extended periods before transplantation. Endod Dent Traumatol 1998;14:79-83.

14. Nethander G. Periodontal conditions of teeth autogenously transplanted by a two-stage technique. J Periodontal Res 1994;29:250-258. doi: 10.1111/j.1600-0765. 1994.tb01219.x.

15. Trope M. Regenerative potential of dental pulp. J Endod 2008;34:S13-S17. doi: 10.1016/j.joen.2008.04.001.

16. Park JH, Tai K, Hayashi D. Tooth autotransplantation as a treatment option: a review. J Clin Pediatr Dent 2010; 35:129-135. 